\title{
The NRC booklet: A group technique for studying word arousal phenomena as a function of $\mathrm{Ss}^{\prime}$
}

\author{
Edward A. Bilodeau, TULANE UNIVERSITY \\ Paul W. Fox, UNIVERSITY OF MINNESOTA
}

\begin{abstract}
Abstraet
A booklet has been designed which can be used with large, intact classes of Ss yet it permits the programming of idiosyncratic training material in experiments on learning and retention.

This note describes new procedures and materials for the study and measurement of word arousal phenomena. The procedures illustrated here employ verbal materials of known strength in the individual's preexperimental associative hierarchy and yet enable $\mathrm{E}$ to take advantage of the methodological and economic benefits of experimentation performed with large, intact classes of Ss. Blending these diverse characteristics into one training and testing instrument is achieved by combining the properties of the verbal booklet developed by Bilodeau et al (1963) with NCR (no carbon required) papers. ${ }^{2}$ The booklet technique is a way of training and testing a group of Ss and is a substitute for the memory drum, flash cards, etc.
\end{abstract}

NCR paper makes use of the reaction between two different chemical coatings and provides sharp, clear reproductions of the S's associative responses on subsequent pages of the booklet when desired. It is technologically superior to carbon paper (no need for carbon inserts, economical, smear resistant, and $\mathrm{S}$ cannot foresee what will happen on later pages). More importantly, NCR paper provides a lightweight, compact, booklet in which every page represents an experimental period, thus facilitating E's page-by-page pacing of an intact class of Ss. By training many Ss simultaneously, it is possible to reduce experimental error contributed by $\mathrm{E}$ variability, differential temporal scheduling, and class sampling. The booklet technique also allows for the inclusion of multiple treatments (or even experiments) within a session or class of Ss and insures a written record of responses for objective scoring when and where $\mathrm{E}$ desires.

Table 1 illustrates one of several applications of the NCR booklet used with an intact class of 400 Ss at

Table 1. Schematic Representation of a Four-Page Booklet Illustrating the Selective Transmission of an S's Continued Associates to "Table."

Page/Event Sequence

Continued Free-

Association

Training-Page A

Training-Page B
Illustration food

Table chair Table leg
Recall of Page B

\author{
Training Page B
}

Psychon. Sci., 1965, Vol. 2. 
Tulane. Each rectangle is a schematic representation of a page, and within pages, only one line is shown in order to conserve space. The E's goal was to (a) assess the individual's associative response hierarchy by having him free associate three times to each stimulus on the first page; (b) train this $\mathrm{S}$ with a list of his idiosyncratic primary associates (see food, in the example) on Page $\mathrm{A}$ and his idiosyncratic secondary responses (such as chair) on Page B; and finally, (c) after a brief interpolated vowel cancelling page (a fifth page not shown), test for the written recall of the Page B material in the presence of the original stimulus word. By applying a colorless desensitizing medium to the blank areas of Pages A and B beforehand or printing black areas upon the pages, it was possible for $\mathrm{E}$ to program which of S's associates would appear for Training on Pages $\mathrm{A}$ and $\mathrm{B}$. Thus, in the illustration, the second associate (chair) is masked on Page A but is reproduced clearly on Page B. The appearance of food on Page A, but not on B, illustrates the masking operation in the opposite direction. The third associate (leg) was not scheduled during Training and has been completely masked. On the recall page of the example in Table 1, S is asked to retrieve the items of Page B in the presence of the stimulus. Food or leg might intrude and might do so more often than for Ss trained on the cultural associates of Table. (Actually, chai $\mathrm{r}$ and food are the primary and secondary, respectively in the cultural norms.) In the experiment described, control Ss were tested for recall in the absence of verbal stimulation, while other Ss were trained with primary and secondary words derived from cultu ral
(Russell \& Jenkins, 1954) rather than idios yncratic norms. The S's employing cultural associates merely copy the words on the first page and by so doing transfer them to Pages A and B. ${ }^{3}$

The simultaneous experimental assessment and regulation of idiosyncratic materials, desirable in research programs which recognize the powerful effects of proacting agents, represents an approach to learning and retention phenomena not readily adaptable to traditional technologies. NCR techniques are suitable for adaptation to a wide range of old (paired-associate, serial learning) and new (stimulated verbal recall, stimulus sampling) tasks requiring single or multiple trial training. They can be used to study any one of a number of communication problems. An application to learning-retention phenomena while measuring and regulating S's preexperimental verbal habits was made here.

\section{Referenees}

BILODEAU, E. A., FOX, P. W., \& BLICK, K. A. Stimulated verbal recall and analysis of sources of recall. J. verbal Learn. verbal Behav., 1963, 2, 422-428.

RUSSELL, W. A., \& JENKINS, J. J. The complete Minnesota norms for responses to 100 words from the Kent-Rosanoff Word Association Test. Tech. Rep. No. 11, 1954, Contract No. N8onr-66216, Office of Naval Research and University of Minnesota.

\section{Notes}

1. This work was supported by contract Nonr-475(10) between Tulane University and the Office of Naval Research.

2. For samples and technical specifications contact the manufacturer, the National Cash Register Co., in Dayton, Ohio or the local office listed in the telephone directory.

3. The approximate cost of performing the experiment described, including all supplies, typing and printing, assembly of booklets, and the actual collection of data was less than $\$ 1.00$ per S. Paper and NCR materials represent but a fraction of this expense. 